\title{
Los índices culturales de Hofstede y su impacto en los estilos de liderazgo en empresas extranjeras que operan en Puerto Rico
}

\author{
Eileen Ortiz Rivera / eortiz@ponce.inter.edu \\ Universidad Interamericana de Puerto Rico \\ Recinto de Ponce
}

\section{RESUMEN:}

El propósito de esta investigación es examinar la relación entre la cultura nacional (las dimensiones culturales) y los estilos de liderazgo que exhiben los gerentes que trabajan en empresas extranjeras que operan en Puerto Rico. Con el propósito de examinar la variable independiente cultura nacional, se utilizaron los índices culturales publicados por Geert Hofstede (1980). Para examinar la variable dependiente estilos de liderazgo, se aplicó el Multifactor Leadership Questionnaire desarrollado por Bass y Avolio (2004). Los resultados del presente estudio muestran que, en general, la cultura nacional explica una variación significativa en el uso de los estilos de liderazgo transaccional y transformacional que exhiben los gerentes que trabajan en las empresas extranjeras que operan en Puerto Rico.

Palabras clave: dimensiones culturales, cultura nacional, estilos de liderazgo, empresas extranjeras.

\section{ABSTRACT:}

This paper examines the relationship between"national culture"(cultural dimensions) and leadership styles displayed by managers in foreign companies operating in Puerto Rico. In this study, the independent variable was "national culture" and the national culture index (Geert Hofstede, 1980) served as indicator. In contrast, leadership styles were the dependent variable measured using the Multifactor Leadership Questionnaire (MLQ) (Bass \& Avolio, 2004). Findings indicate that, overall, "national culture" resulted as a significant variation in transactional and transformational leadership styles employed by managers working in foreign companies operating in Puerto Rico.

Keywords: cultural dimensions, national culture, leadership styles, foreign companies. 


\section{INTRODUCCIÓN}

La evolución de las economías mundiales ha estimulado el desarrollo de las relaciones comerciales globales. La globalización de la fuerza trabajadora, la expansión de las multinacionales y la exposición de las compañías locales hacia un mundo más competitivo, han provocado que las firmas evalúen más a fondo cómo la cultura nacional de un país interviene en los estilos de liderazgo de los gerentes (Zagorsek, 2005). El liderazgo ha sido una de las preocupaciones más antiguas a nivel mundial. Éste puede manifestarse en todos los grupos de personas, sin importar la geografía, la cultura o la nacionalidad (House, Hanges, Ruiz-Quintanilla, Dorfman, Javidan, Dickson \& Gupta, 1999). Desde el punto de vista organizacional, el liderazgo a menudo juega un rol importante y es uno de los principales determinantes del éxito o el fracaso de una compañía (Bass, 1990). Estudios organizacionales han demostrado que el liderazgo de los ejecutivos cuenta con más de un $45 \%$ de la ejecución total de la organización (Day \& Lord, 1988). Investigaciones realizadas por Howell y Costley (2001) y Schriesheim y Neider (1996) indican que el liderazgo hace una gran diferencia en la ejecución de las tareas y satisfacción de los empleados con su trabajo.

En la mayoría de las circunstancias, las empresas multinacionales envían inicialmente gerentes del país de origen (expatriados) a sus subsidiarias en el extranjero. Posteriormente van colocando más ejecutivos del país anfitrión en los puestos gerenciales conforme éstos adquieren experiencia (Hill, 2007). Hay estudios que han revelado que solamente el $30 \%$ de los gerentes del país de origen (expatriados) recibieron alguna capacitación antes de su partida hacia el país extranjero (GMAC GRS, Survey 2005). Dada esta situación, los gerenciales sufren de problemas de adaptación en sus prácticas gerenciales y en su entorno personal, lo que repercute en una disminución en el desempeño y en una ausencia de liderazgo organizacional. Estudios han revelado que la adaptación cultural en las prácticas administrativas propicia un ambiente agradable de trabajo (Hofstede, 1980), en el que tanto los gerenciales como los empleados pueden desempeñarse mejor y alcanzar un nivel de productividad óptimo, logrando a su vez el éxito de la empresa bajo su industria y en la economía local. 
Los cambios demográficos en la fuerza laboral implican que los gerentes deben demostrar mayor interés por comprender las complejidades asociadas con la supervisión de los empleados de diferentes culturas (Thomas \& Au, 2002). En este nuevo contexto surgen estudios que examinan la relación entre la cultura y los estilos de liderazgo transaccional y transformacional (Ardichvili \& Kuchinke, 2002; Zagorsek, 2005). Dentro de la gerencia internacional, el estilo de liderazgo transaccional es el clásico y el más utilizado dentro de las organizaciones internacionales (Cullen \& Parboteeah, 2005), ya que guía y motiva a sus seguidores a que exhiban una serie de comportamientos deseados a fines de lograr las metas establecidas aclarando los roles y las tareas, los cuales se refuerza mediante un sistema de recompensas y/o castigos (Robbins, 2004). Junto con el liderazgo transaccional surge el liderazgo transformacional, el cual es distintivo pero no mutuamente excluyente, en el que los seguidores sienten confianza, admiración, lealtad y respeto hacia el líder (Yukl, 2010).

Estudios clásicos como los realizados por Hofstede (2005) y Rodrigues (1990) sugieren la existencia de ciertas relaciones entre las dimensiones culturales y los estilos de liderazgo que emplean los supervisores. Por ejemplo, los países donde existen altos índices de distancia de poder y de evasión a la incertidumbre, están asociados con comportamientos autocráticos relacionados con el estilo de liderazgo de tipo transaccional. Ampliando esa misma línea de análisis, el propósito de esta investigación es examinar la relación entre la cultura nacional (las dimensiones culturales) y los estilos de liderazgo que exhiben los gerentes que trabajan en empresas extranjeras operando en Puerto Rico.

\section{MARCO TEÓRICO E HIPÓTESIS}

La literatura académica indica que no existe una fórmula que identifique cómo ser un líder en cada contexto nacional (Cullen $\&$ Parboteeah, 2005). Sin embargo existen estudios, como los llevados a cabo por Geert Hofstede, que presentan algunas recomendaciones generales. En el estudio clásico de Hofstede (1980) sobre el impacto de las culturas nacionales y su relación con las actitudes 
y los valores de los empleados, éste argumenta que muchas de las diferencias en la motivación de los empleados, los estilos gerenciales y las estructuras organizacionales encontradas alrededor del mundo están relacionadas con las diferencias culturales. Originalmente, el autor comparó los factores culturales de cuarenta países, tomando como muestra la corporación multinacional IBM. Luego continuó explorando las diferencias culturales existentes entre los miembros de hasta 72 países y regiones. Hofstede (1980) define cultura como la "programación mental colectiva de las personas en diferentes ambientes sociales".

En su estudio, Hofstede (1980) reveló la existencia de cuatro dimensiones culturales que se midieron utilizando el cuestionario llamado Values Survey Module (el VSM, por sus siglas en inglés). Estas cuatro dimensiones culturales son las siguientes: la distancia de poder, el individualismo, la masculinidad y la evasión a la incertidumbre. Hofstede desarrolló índices que reflejan las características culturales nacionales o dimensiones culturales de un país. Con estos índices culturales, las empresas multinacionales estarían mejor capacitadas para poder manejar las diferencias entre culturas en sus negocios globales (Hofstede, 2005).

Hofstede (2005) define distancia de poder como el grado en que los miembros con menos poder en las instituciones y organizaciones de un país esperan y aceptan que el poder esté distribuido de manera desigual. El autor descubrió que muchos países latinoamericanos y asiáticos se caracterizan por una gran distancia de poder. En estos países, los gerentes tienen la tendencia a tomar decisiones autocráticamente, la gran mayoría de las organizaciones son paternalistas y los subordinados aparentan obedecer las órdenes sin cuestionarlas (Hofstede, 2005).

El individualismo es característico de sociedades donde las personas se ocupan de sí mismas y de su familia más próxima (Hofstede, 2005). El colectivismo, por el contrario, es característico de sociedades en las que las personas se integran desde su nacimiento en grupos fuertes y cohesionados que continúan protegiéndolas toda la vida a cambio de lealtad (Hofstede, 2005). Según el autor, en los países con un alto grado de individualismo, se le da mucha importancia a la iniciativa y al logro individual y se estimula (motiva) a la gente a 
tomar decisiones personales sin contar con el respaldo del grupo. Por el contrario, en países con un nivel bajo de individualismo, se le otorga mucha importancia a la toma de decisiones en grupo (Hofstede, 2005).

La dimensión de masculinidad es el grado en que los valores dominantes de una sociedad son: el éxito, el dinero y los bienes materiales (Hofstede, 2005). En los países con un alto nivel de masculinidad se aprecian mucho el reconocimiento, el progreso y los retos. En estas culturas se tiende a favorecer a las empresas con un gran crecimiento económico. Hofstede midió esta dimensión en contraste con la femineidad, que es el grado en que los valores dominantes son el interés por los demás y por la calidad de vida. En los países con índices de femineidad, el ambiente de trabajo es agradable. El logro se define por los contactos humanos que se tenga.

Hofstede (2005) define la dimensión cultural evasión de la incertidumbre como la medida en que los miembros de una cultura se sienten amenazados frente a situaciones desconocidas, inciertas o incómodas antes situaciones ambiguas o arriesgadas. Los países con una tendencia hacia la evasión de la incertidumbre, como Japón, tratan de disminuir el riesgo y de crear sistemas y mecanismos para afrontar la ambigüedad (Hofstede, 2005). A su vez, tienden a formalizar las actividades organizacionales y enfatizan el uso de normas y reglas para asegurarse que la gente sepa lo que debe hacer. Por el contrario, en países como Canadá, en donde están menos inclinados a evadir la incertidumbre, las actividades organizacionales son menos estructuradas y están dirigidas a estimular a las personas a asumir riesgos. Como consecuencia, las personas muestran menos estrés y recurren frecuentemente a la iniciativa personal y al ingenio para hacer las cosas (Hofstede, 2005).

Cabe señalar que las culturas personales y nacionales se derivan de los valores únicos de la gente y se proyectan al estilo o papel del liderazgo gerencial y a su vez al desempeño de las empresas (Byrne \& Bradley, 2007). Dentro de los diversos tipos de liderazgo podemos mencionar dos estilos fundamentales -el transformador y el transaccional- que están acorde con las nuevas tendencias del siglo y con el desarrollo de la globalización de los negocios. El liderazgo transformacional cambia el nivel de concienciación de sus seguido- 
res con respecto a lo que es importante, los mueve a que se vean a sí mismos y hacia las oportunidades y los retos del medioambiente de una manera nueva (Bass \& Avolio, 2004). En contraste, el liderazgo transaccional involucra un proceso de intercambio que puede resultar en que el empleado cumpla con las peticiones del líder, pero probablemente no genere entusiasmo y compromiso con los objetivos del trabajo (Yukl, 2010). Según este autor la esencia distintiva entre los estilos de liderazgo transformacional y transaccional se define en término del comportamiento de los líderes para influenciar a los seguidores y el efecto del comportamiento de éstos en los seguidores.

Bhaskaran \& Sukumaran (2007) llevaron a cabo un estudio en el que se demostró que la cultura nacional tiene mayor efecto sobre los empleados que el efecto que pueda ejercer la propia cultura organizacional. El programa Global Leadership and Organizational Behavior Effectiveness (GLOBE) analizó los comportamientos interculturales de liderazgo exhibidos por más de 18,000 gerentes ubicados en 62 países (House, Chhokar \& Brodbeck, 2004). De hecho, estudios menos abarcadores han encontrado que la etnicidad influye en la percepción del empleado respecto a la orientación cultural y el estilo administrativo o gerencial que exhiben los gerentes (Vargas, 2005). De igual manera, un estudio realizado entre 1,015 dueños-gerentes de pequeñas empresas en 12 países latinoamericanos (Argentina, Bolivia, Brasil, Chile, Colombia, Ecuador, México, Paraguay, Perú, Puerto Rico, Uruguay y Venezuela) encontró diferencias en la importancia que se les atribuye a los valores culturales (Lenartowicz \& Johnson, 2003). Considerando los hallazgos previos, se planteó la siguiente hipótesis:

$\mathbf{H}_{1}$ : Existe una relación estadísticamente significativa entre la cultura nacional y los estilos de liderazgo de los gerentes de empresas extranjeras que operan en Puerto Rico.

Lebrón-Rolón (2008) examinó si había alguna relación entre los estilos de liderazgo y la cultura entre gerentes de Puerto Rico y República Dominicana. El estudio encontró significancia en que los gerentes dominicanos presentan puntuaciones altas en las dimensiones relacionadas con distancia de poder y con relación a la dimensión de masculinidad. Éstos exhibieron un estilo gerencial paternalista. 
De acuerdo con la literatura, el estilo gerencial paternalista tiene una tendencia similar al estilo autocrático, es decir, se dicta el trabajo y se centraliza la toma de decisiones (Robbins \& Coulter, 2008). El estilo gerencial y las características culturales que exhibieron los gerentes dominicanos se alineaban con el estilo de liderazgo transaccional (Lebrón-Rolón, 2008). Por su parte, Elenkov (1998) también afirmó que en culturas con un alto grado de evasión a la incertidumbre y gran distancia de poder (Francia), los líderes emplean más el estilo de liderazgo transaccional. Estos hallazgos junto con los planteamientos de Hofstede, llevan a plantear nuestra segunda hipótesis:

$\mathbf{H}_{2}$ : Los gerentes de países con alto índice en distancia de poder, individualismo, masculinidad y evasión a la incertidumbre, presentan mayor relación respecto al estilo de liderazgo transaccional.

Jung, Bass \& Soski (1995) encontraron que el liderazgo transformacional es más efectivo en culturas colectivistas (China), ya que este tipo de liderazgo favorece la habilidad para adaptarse, resolver problemas y mejorar el desempeño organizacional tomando en consideración la participación de los empleados (subordinados). Zagorsek (2005) exploró la relación entre cultura y el liderazgo transformacional en una muestra de 804 estudiantes graduados (MBA) de seis países (Estados Unidos, Nigeria, Eslovenia, Corea, India y Argentina) en cinco continentes y encontró que la cultura nacional, aunque tiene poco impacto, resulta significativa en su influencia en la práctica del liderazgo transformacional. Thomany (2009) no examinó, explícitamente, las dimensiones culturales de Hofstede, pero sí destacó la manifestación de la preocupación por las relaciones de los integrantes de la empresa y la disponibilidad de los ejecutivos latinoamericanos para atender asuntos de las empresas junto a los empleados, características que se alinean a las bajos índices de distancia de poder y masculinidad (feminidad).

En el mencionado estudio de Lebrón-Rolón (2008), también se encontró significancia en que los gerentes puertorriqueños presentan puntuaciones bajas en distancia de poder y en masculinidad y tienen un estilo gerencial democrático. De acuerdo con la literatura, el estilo gerencial democrático involucra a los subordinados, delega 
autoridad y promueve la participación de los empleados (Robbins \& Coulter, 2008). Dichas características parean con el estilo de liderazgo transformacional, por lo que se puede interpretar que LebrónRolón (2008) encontró relación entre los gerentes puertorriqueños con bajo índice de distancia de poder y masculinidad y el estilo de liderazgo transformacional. De este modo, se plantea nuestra tercera hipótesis:

$\mathbf{H}_{3}$ : Los gerentes de países con índices bajos en distancia de poder, individualismo, masculinidady evasión de la incertidumbre presentan mayor relación respecto al estilo de liderazgo transformacional.

En un estudio llevado a cabo entre estudiantes graduados de varios países (Nigeria, Estados Unidos y Eslovenia), Zagorsek, Jaklic y Stough (2004) encontraron que las diferencias entre el género y el estilo de liderazgo dependen del país de origen. A su vez, Woo, Wilson y Liu (2001) demostraron que el factor género interviene en los procesos de las negociaciones entre China y los Estados Unidos cuando éstos discuten o negocian aspectos tales como asuntos comerciales. Por otra parte, en un estudio realizado en 1995, Kustin y Jones encontraron que los CEO japoneses bien preparados prefieren un estilo de liderazgo autocrático y corporativo (se alinean con el estilo de liderazgo transaccional). A base de los estudios, se establece la cuarta hipótesis:

$\mathbf{H}_{4}$ : Hay diferencia estadísticamente significativa en género, edad, tipo de industria, preparación académica, años de experiencia trabajando en el puesto como gerentes de empresas extranjeras que operan en Puerto Rico y los estilos de liderazgo.

Otras investigaciones llevadas a cabo en este campo han encontrado que las subsidiarias reciben una influencia significativa de las oficinas centrales en el país de origen (Kustin \& Jones, 1995). Esto sugiere que las empresas que operan en países extranjeros emplean estilos administrativos que guían a sus seguidores en la dirección de las metas establecidas por las oficinas centrales aclarando los roles y las tareas (Robbins, 2004), y que a su vez están alineadas con el estilo de liderazgo transaccional. Entonces, se plantea la quinta hipótesis: 
$\mathbf{H}_{\mathbf{5}}$ : En las empresas extranjeras que operan en Puerto Rico predomina el estilo de liderazgo transaccional.

\section{Metodología}

\section{MUESTRA Y COLECCIÓN DE DATOS}

De acuerdo con el propósito y los objetivos del estudio, la población a examinarse fueron las empresas extranjeras con operaciones y/o presencia en Puerto Rico (empresas cuyas oficinas centrales se encuentran radicadas fuera de Puerto Rico). Se recurrió a la Compañía de Fomento Industrial de Puerto Rico (Puerto Rico Industrial Development Company, PRIDCO, por sus siglas en inglés), la cual suministró una base de datos en la que se identificaron un total de 67 empresas extranjeras (excluyendo las empresas estadounidenses) en diferentes áreas de la industria de manufactura y venta de productos. ${ }^{1}$ A base de la población identificada se realizó un muestreo aleatorio que resultó en 52 empresas extranjeras. ${ }^{2}$ En octubre se enviaron los cuestionarios a los ejecutivos de mayor jerarquía, o en su defecto, a los empleados gerenciales de dichas empresas. Se recibieron 32 cuestionarios debidamente cumplimentados, lo que representa un $61 \%$ de tasa de respuesta.

\section{INSTRUMENTO DE MEDICIÓN}

Para examinar la variable independiente "cultura nacional" (las dimensiones culturales) se utilizaron como datos secundarios los índices culturales publicados por Geert Hofstede (1980) y los índices culturales para Puerto Rico publicados por Irma L. Alvarado Zayas (2005). Para examinar la variable dependiente estilos de liderazgo transaccional y transformacional se utilizó el Multifactor Leadership Questionnaire (MLQ, por sus siglas en inglés) desarrollado por Bass y

1 El autor deliberadamente excluyó las empresas estadounidenses que operan en Puerto Rico, debido a la convergencia de la cultura nacional y organizacional de dicho país en Puerto Rico, como resultado de la relación política histórica entre los mencionados países.

${ }^{2}$ Las empresas extranjeras que operan en Puerto Rico objeto de este estudio tenían sus oficinas centrales ubicadas en los siguientes países: Venezuela, Perú, Francia, Japón, Suecia, Austria, España, Colombia y República Dominicana. 
Avolio (2004). El MLQ ha sido extensamente empleado en aproximadamente trescientos programas de investigación, disertaciones doctorales y tesis de maestría alrededor del mundo desde el 1995 al 2004. Estudios previos que han examinado la cultura nacional y los estilos de liderazgo (Ardichvili \& Kuchinke, 2002), han utilizado específicamente el MLQ 5X (versión corta), el cual es la versión más reciente para medir el comportamiento de liderazgo transaccional y transformacional en los gerentes. La versión del instrumento MLQ5X se ha traducido a varios idiomas, entre ellos el español, y ha sido validado y utilizado en proyectos de investigación académica en Puerto Rico (Vélez, 2008; Vázquez, 2007; López, 2006).

El MLQ5X (versión corta) contiene un total de 45 preguntas (reactivos); las primeras 36 preguntas miden los estilos de liderazgo transaccional (y sus cuatro dimensiones) y el estilo de liderazgo transformacional (y sus cinco dimensiones), y las últimas nueve (9) preguntas miden efectividad, esfuerzo adicional (extra effort) y satisfacción. ${ }^{3}$

Todas las 45 preguntas (reactivos) en el MLQ 5X (versión corta) para medir los estilos de liderazgo transaccional ( $\mathrm{y}$ sus dimensiones) y transformacional (y sus dimensiones), se examinaron en una escala Likert de cinco puntos (five-point scale). A los encuestados se les pidió que valoraran cuán frecuentemente cada aseveración (reactivo) describe o se alinea con el comportamiento del líder.

El cuestionario también auscultó en datos las personas encuestadas, específicamente: género, edad, tipo de industria, preparación académica y años de experiencia en el puesto. Todos estos datos ofrecieron información relevante para delinear un perfil de los encuestados en el estudio y examinar su relación con los estilos de liderazgo (hipótesis cuatro del estudio).

\section{Resultados}

Las relaciones entre la cultura nacional (variable independiente) y los estilos de liderazgo (variable dependiente) planteadas en las hipótesis de trabajo se examinaron por medio de análisis de regresión. La Tabla 1 presenta los resultados de dichos análisis.

3 Para el del MLQ se obtuvo el permiso de los autores Bass y Avolio. 


\begin{tabular}{|c|c|c|c|c|}
\hline 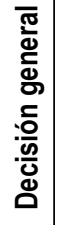 & \multicolumn{3}{|l|}{ 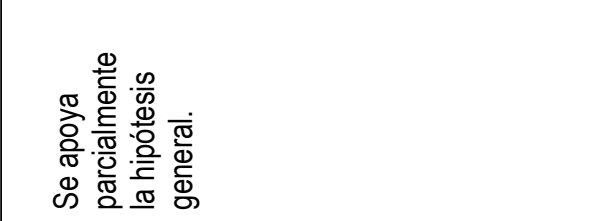 } & 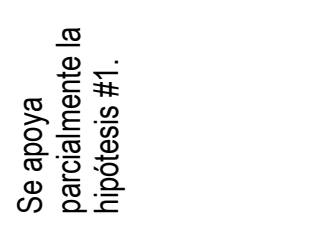 \\
\hline 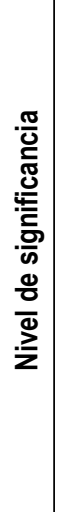 & 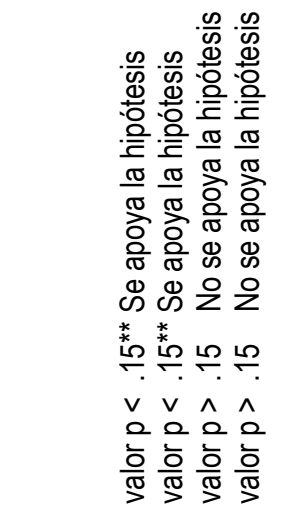 & & 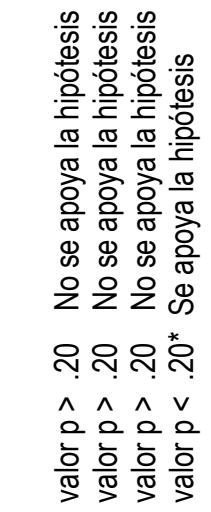 & 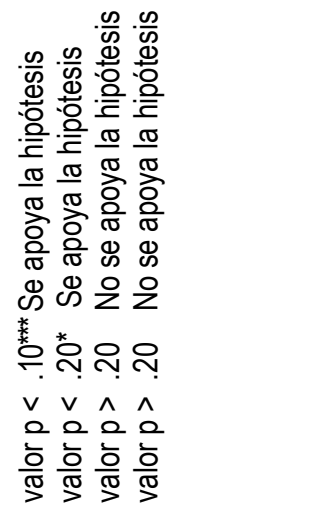 \\
\hline 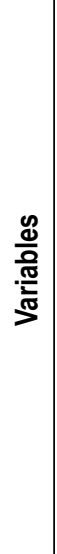 & 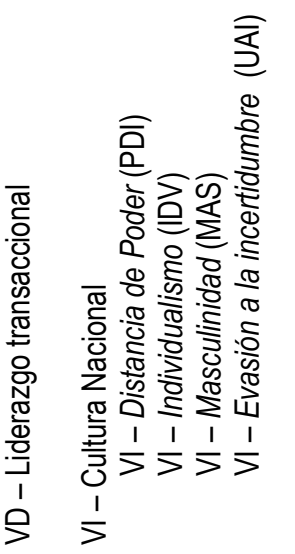 & 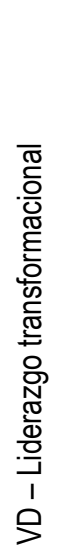 & 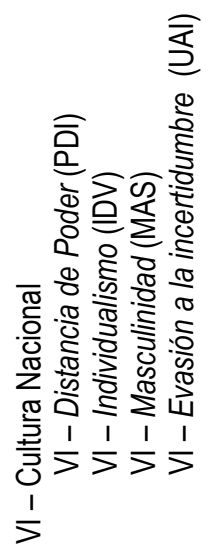 & 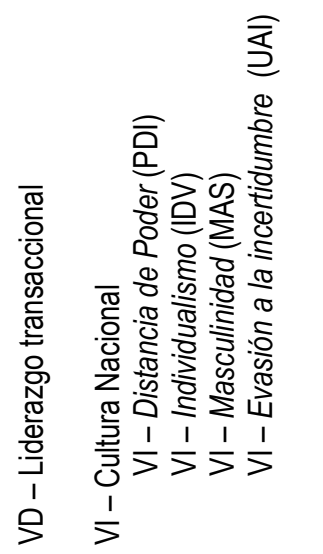 \\
\hline 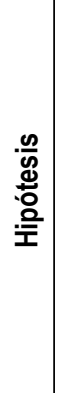 & 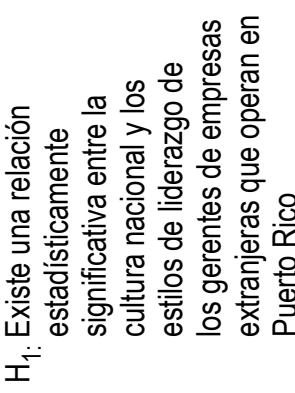 & & & 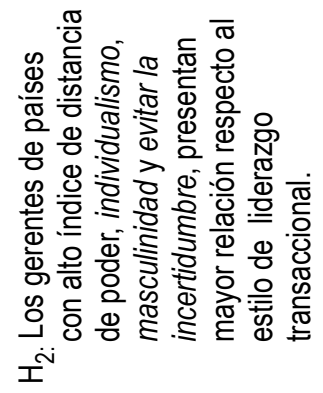 \\
\hline
\end{tabular}




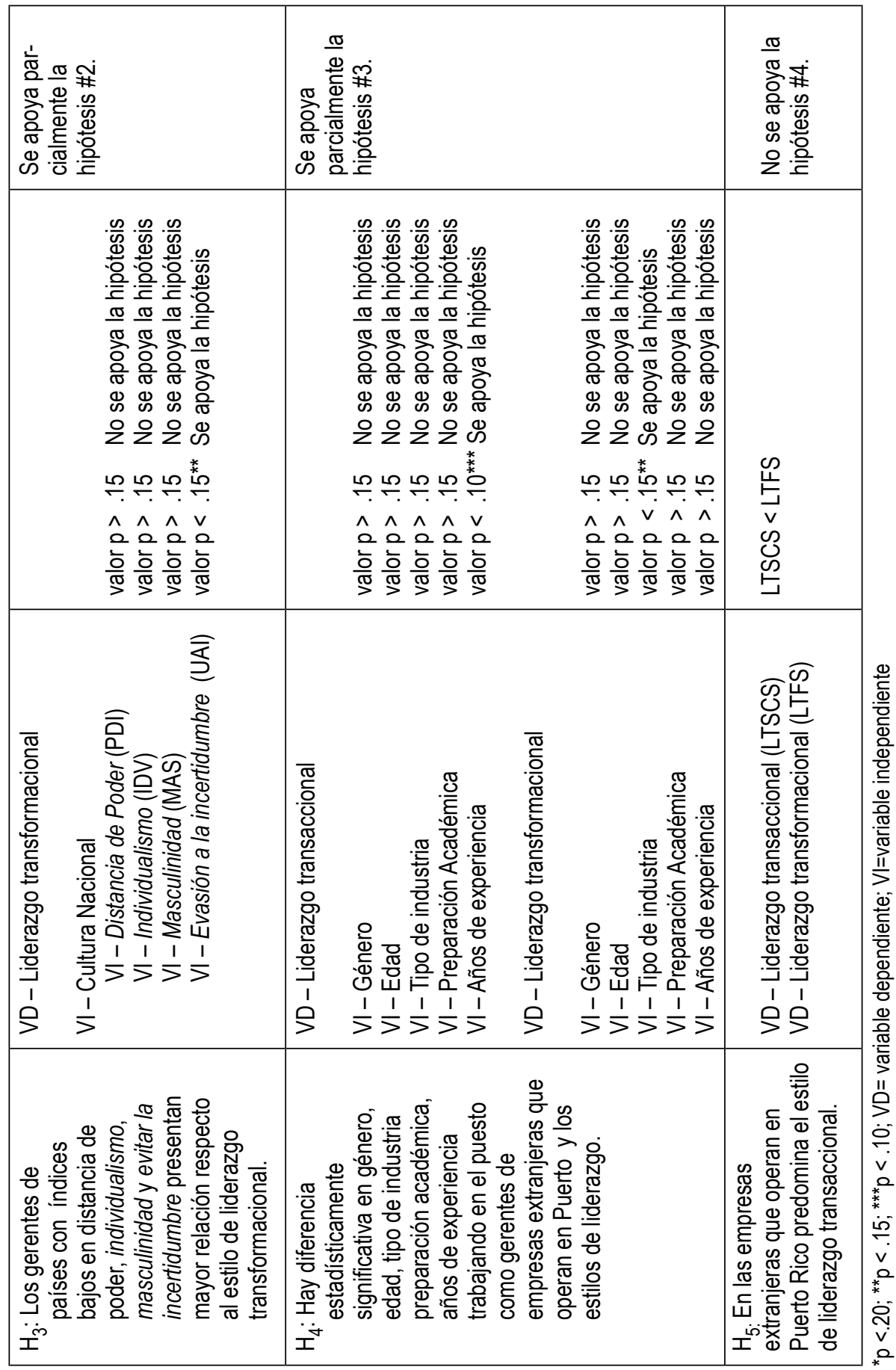


En la hipótesis $1\left(\mathrm{H}_{1}\right)$ se encontró una relación estadísticamente significativa entre las sub-dimensiones culturales de distancia de poder (PDI) e individualismo (IDV) con el liderazgo transaccional a un nivel de significancia máxima de .15. También se encontró relación estadísticamente significativa entre la sub-dimensión cultural de evasión de la incertidumbre (UAI) con el liderazgo transformacional a un nivel de significancia máxima de .20 . Se encontró relación estadísticamente en dos de las cuatro sub-dimensiones culturales respecto al liderazgo transaccional y en una sub-dimensión cultural respecto al liderazgo transaccional. Por lo tanto, la hipótesis 1 del estudio con respecto a la existencia de una relación estadísticamente significativa entre la cultura nacional y los estilos de liderazgo de los gerentes de empresas extranjeras que operan en Puerto Rico se apoya parcialmente.

A partir de la hipótesis $1\left(\mathrm{H}_{1}\right)$, se especificaron unos modelos de regresión lineal para las hipótesis $2\left(\mathrm{H}_{2}\right)$ y $3\left(\mathrm{H}_{3}\right)$, en las que se encontraron las mismas relaciones de las sub-dimensiones de distancia de poder (PDI) e individualismo (IDV) con el liderazgo transaccional y la sub-dimensión de evasión de la incertidumbre (UAI) con el liderazgo transformacional, pero a diferentes grados de significancia. A base de dichos datos, se apoyaron parcialmente las hipótesis 2 y 3 del estudio.

En la hipótesis $4\left(\mathrm{H}_{4}\right)$ se encontró diferencia estadísticamente significativa en la variable tipo de industria con el liderazgo transformacional. De igual manera, se encontró diferencia estadísticamente significativa entre la variable demográfica años de experiencia con el liderazgo transaccional. La diferencia estadísticamente significativa de dos de las cinco variables demográficas respecto a los estilos de liderazgo apoya parcialmente la hipótesis 4 del presente estudio.

Finalmente, el liderazgo transformacional mostró una media más alta que el liderazgo transaccional, lo que indica que el liderazgo transformacional predomina en las empresas extranjeras encuestadas. Estos datos no apoyaron la hipótesis 5, que planteaba que el liderazgo transaccional era el predominante en las empresas extranjeras que operan en Puerto Rico.

En la Tabla 2 se presenta un resumen de las estadísticas descriptivas de los datos demográficos de los encuestados, que permite delinear un perfil de las empresas y/o personal encuestados. 
Tabla 2: Resumen de las estadísticas descriptivas de los datos demográficos de las empresas y los encuestados

\begin{tabular}{lccc}
\hline Datos demográficos & Media & Mediana & Moda \\
\hline Área de negocio & --- & -- & Manufactura \\
Título o posición & -- & --- & $\begin{array}{l}\text { Ejecutivo } \\
\text { principal }\end{array}$ \\
Edad & 47.66 & 50 & 40 \\
Género & --- & --- & Masculino \\
Nivel de educación & 17.5 & 18 & 18 \\
$\begin{array}{l}\text { Años de experiencia } \\
\text { en el puesto }\end{array}$ & 11.8 & 13 & 21 \\
$\begin{array}{l}\text { Nacionalidad } \\
\text { País de nacimiento }\end{array}$ & --- & --- & $\begin{array}{c}\text { Americano } \\
\text { Puerto Rico }\end{array}$ \\
\hline
\end{tabular}

La interpretación de los datos que se presentan en la Tabla 2, permite delinear un perfil de las empresas y/o personal encuestados. Por ejemplo, los gerenciales y/o ejecutivos de mayor jerarquía encuestados reflejaron una edad promedio de 48 años, con estudios graduados (18 años de estudios) y aproximadamente 12 años de experiencia en la industria en la que se desempeña su empresa. Por otro lado, la mayoría de los encuestados informó que poseía la nacionalidad americana (estadounidense). Puerto Rico resultó ser el país de nacimiento que se reportó con más frecuencia entre los encuestados en el presente estudio. Dichos datos nos indican que estas empresas reclutan, seleccionan y confían sus operaciones en la Isla a gerentes (locales/oriundos de Puerto Rico) maduros, bien preparados académicamente y experimentados (con más de 10 años de experiencia en la industria en la que se desempeña la empresa).

\section{Discusión}

En la literatura existen estudios que nos presentan resultados mixtos respecto a la relación entre los aspectos propios de la cultura de un individuo y sus estilos gerenciales. Algunos estudios encuentran que existe una convergencia cultural y de prácticas 
gerenciales entre países con cercanía geográfica e histórica (Moro, 2005; Punnet, Dick-Forbe \& Robinson, 2006), mientras que otros presentan una divergencia o diferencias estadísticamente significativas en la importancia de los valores culturales y los estilos que utilizan los gerentes al momento de negociar en países que teóricamente se consideran culturalmente homogéneos (Lenartowicz \& Johnson, 2003; Niedziolek, 2005). Este estudio demuestra que sólo algunas de las dimensiones culturales de Hofstede (2005) tienen relación con diferentes niveles de significancia con los estilos de liderazgo que exhiben los gerentes de empresas extranjeras que operan en Puerto Rico. Tales hallazgos sugieren que, aunque puede estar correcto presumir que existe una relación significativa entre los estilos de liderazgo que utilizan los supervisores basados en la cultura nacional, la importancia de dicha relación en las prácticas gerenciales observadas por los gerentes en las empresas estudiadas puede variar dependiendo del país de donde proviene la empresa.

Algunos estudios tienden a apoyar el planteamiento de nuestra hipótesis 2 de altos índices de distancia de poder e individualismo y estilos de liderazgo transaccional (Lebrón-Rolón, 2008). No obstante, otros estudios comparten nuestros hallazgos de una relación contraria o inversa, es decir, a menor valor de distancia de poder e individualismo, se observa un mayor valor de índice de liderazgo transaccional (Vargas, 2005). De acuerdo con Hill (2007), los gerentes en grupos culturales con un índice bajo o moderado en la sudimensión distancia de poder (PDI) consultan a los subordinados (menos individualistas) antes de tomar una decisión en la organización. Esta dinámica puede asociarse con la sub-dimensión de recompensa contingente de liderazgo transaccional en la que el gerente aclara las metas y los objetivos de la organización y provee reconocimiento una vez se alcanzan las metas, lo que resulta en individuos o empleados que logran los niveles de desempeño esperados (Bass \& Avolio, 2000). Esto puede ser propio de una empresa extranjera que busca eficacia y eficiencia en sus operaciones en el extranjero como es el caso de la población del presente estudio. Un análisis cualitativo de los niveles de las sub-dimensiones culturales de Hofstede y el estilo de liderazgo transaccional sugiere que, además de la cultura nacional, otros aspectos (como la cultura organizacional) también podrían intervenir 
en los estilos de liderazgo adoptados por los gerentes de empresas extranjeras que operan en Puerto Rico.

El presente estudio encontró que sólo la sub-dimensión cultural relacionada con la evasión de la incertidumbre (UAI) tiene una relación positiva estadísticamente significativa con el estilo de liderazgo transformacional. Se ha documentado la influencia que ejercen las oficinas centrales en la toma de decisiones de sus subsidiarias y cómo este hecho se refleja en los estilos de liderazgo exhibidos por sus gerentes (Kustin \& Jones, 1995). Los hallazgos del presente estudio sugieren que el nivel de predictividad de la cultura nacional, utilizando las dimensiones culturales de Hofstede en el estilo de liderazgo transformacional en los gerentes de empresas extranjeras que operan en Puerto Rico es parcial y se puede complementar al considerar otras variables tales como la cultura organizacional de la empresa..

$\mathrm{Al}$ examinar las variables demográficas del estudio, se encontraron diferencias estadísticamente significativas entre los tipos de industria y los años de experiencia que poseen los gerentes en el puesto respecto al estilo de liderazgo que éstos exhiben en las empresas. Estos hallazgos sugieren que, además de la cultura nacional (medida por las sub-dimensiones de Hofstede), las variables relacionadas con la organización y el personal dentro de ésta pueden aportar, en mayor o menor medida, un poder predictivo sobre la utilización de los estilos de liderazgo que tiende a exhibir los gerentes de empresas extranjeras que operan en Puerto Rico.

A través de un análisis descriptivo que compara las medias de los estilos de liderazgo transaccional y transformacional, se encontró que el estilo de liderazgo que predomina en las empresas extranjeras que operan en Puerto Rico es el liderazgo transformacional. Esto se alinea y confirma los hallazgos de varios estudios realizados donde se ha examinando los estilos de liderazgo en las organizaciones educativas en Puerto Rico (López, 2006; Rentas-Rivera, 2009; Vázquez, 2007; Vélez-Santiago, 2008). También se alinea con los hallazgos de Lebrón-Rolón (2008), quien encontró que los gerentes de empresas puertorriqueñas presentan un estilo gerencial democrático, cuyas características se asocian con el estilo de liderazgo transformacional. Tales hallazgos sugieren que en Puerto Rico los gerentes de las em- 
presas extranjeras tienden a utilizar el estilo de liderazgo transformacional en sus funciones administrativas.

\section{ConcLusión}

Los resultados del presente estudio demuestran que, en general, la cultura nacional (medida por los índices culturales de Hofstede) explica una variación significativa en el uso de los estilos de liderazgo transaccional y transformacional que exhiben los gerentes de empresas extranjeras que operan en Puerto Rico. En estudios realizados sobre la práctica de liderazgo transformacional en seis países se encontró que la cultura, como única variable independiente, explica el $10 \%$ de la variación en el uso de prácticas de liderazgo, mientras que, de otro modo, sólo explica el 5\% (Zagorsek, 2005). En estudios entre 63 países, GLOBE encontró que las dimensiones culturales (valores culturales) a nivel social y organizacional explican el $10 \%, 26 \%, 7 \%$ y $12 \%$ de toda la variación organizacional de los cuatro estilos de liderazgo culturalmente aprobados por la organización (House, Hanges, Ruiz-Quintanilla, Dorfman, Javidan, Dickson \& Gupta, 1999).

La influencia en diferentes niveles de significancia de la cultura nacional y los estilos de liderazgo puede entenderse, dado que el liderazgo como fenómeno puede tener numerosos antecedentes causales. El liderazgo puede considerarse como un proceso que guía e influye en un grupo para alcanzar unas metas (Robbins \& Coulter, 2008). Esto sugiere que existen muchas variables no incluidas en el presente estudio que pueden determinar o influir en el uso de un estilo o práctica de liderazgo en específico en los gerentes. Por ejemplo, las características del líder (personalidad, capacidades, motivaciones, valores y creencias), las características de los subordinados (sus personalidades, necesidades y expectativas, destrezas y experiencia y compromiso con la empresa) y las características de la situación (tipo y estructura de la organización, ambiente específico de la industria, la cultura nacional y organizacional) pueden incidir en el proceso de adopción y/o práctica de un estilo de liderazgo particular y en los resultados que se deriven de esto (Zagorsek, 2005). La cultura nacional puede considerarse como una de las variables 
influyentes, aunque se puede argumentar que otras variables, tales como los valores y las expectativas del gerente y los subordinados y el tipo de cultura organizacional, pueden ejercer algún tipo de influencia. Cuando se toman en consideración las complicaciones que pueden ocurrir en una investigación exploratoria, resulta importante el hallazgo de relación (a diferentes niveles de significancia) entre algunas de las dimensiones culturales de Hofstede y las variables demográficas (años de experiencia y tipo de industria) con respecto a los estilos de liderazgo de los gerentes en las empresas extranjeras que operan en Puerto Rico. Por lo tanto, podemos inferir que la cultura nacional tiene importancia, aunque su impacto en los estilos de liderazgo pueda variar como resultado de otras posibles variables.

Puesto que las relaciones entre el liderazgo y la cultura nacional en el presente estudio son parciales, concluimos que se deben continuar las investigaciones en otros niveles. Por ejemplo, examinar el impacto de la cultura organizacional y los estilos de liderazgos, ya que puede jugar un papel más importante en la formación del comportamiento de los gerentes, que la cultura a nivel de país (Ardichvili \& Kuchinke, 2002).

\section{CONTRIBUCIÓN E IMPLICACIONES}

El presente estudio tiene un valor significativo por sus implicaciones teóricas y prácticas para un mejor entendimiento y funcionamiento en el ambiente laboral entre los gerentes y empleados locales en las empresas extranjeras que operan en Puerto Rico. Es el primer estudio empírico que se lleva a cabo para medir la relación entre la cultura nacional y los estilos de liderazgo que exhiben los gerentes en sus funciones administrativas en dichas empresas. Esto constituye un adelanto y un punto de partida para llenar el vacío existente en la literatura actual sobre los choques culturales entre los estilos de liderazgos que exhiben los gerenciales y los empleados locales que trabajan en empresas extranjeras que operan en Puerto Rico.

Además, por primera vez, se confirma la relación o influencia que ejerce la cultura nacional sobre los estilos de liderazgo que exhiben los gerentes de las empresas extranjeras que operan en la Isla, 
utilizando los índices culturales de Hofstede. Un gerente extranjero con interés en adaptar sus prácticas gerenciales (los estilos de liderazgo) en escenarios laborales puertorriqueños, puede utilizar de referencia los hallazgos de la presente investigación para lograr una mayor efectividad en el uso de los recursos en su empresa y un mejor desempeño organizacional.

Se creó un perfil de los gerentes y de las empresas extranjeras que operan en Puerto Rico y se documenta el hecho que los gerentes en dichas empresas exhiben un estilo de liderazgo transformacional en sus funciones administrativas. Este hallazgo rompe con los posibles estereotipos que se han formado sobre la clase gerencial en empresas extranjeras que operan en Puerto Rico y las posibles motivaciones de las mismas en establecerse en la Isla. Otra contribución muy valiosa es que el estudio no se limitó a un sector industrial ni geográfico en Puerto Rico, lo que permite una generalización real de los hallazgos.

A diferencia de otros estudios llevados a cabo en el contexto internacional, esta investigación se destaca y es valiosa debido a su aplicación en Puerto Rico (representando el Caribe), específicamente en empresas extranjeras (excluyendo las continentales) que operan en la Isla. 


\section{RefERENCIAS}

Alvarado Zayas, I. L. (2005). Puerto Rico: Clasificación cultural del modelo de Hofstede y su impacto en la adaptación cultural y desempeño organizacional de las empresas multinacionales. Disertación Doctoral, Universidad Interamericana de Puerto Rico.

Ardichvili, A., \& Kuchinke, K. P. (2002). Leadership styles and cultural values among managers and subordinates: A comparative study of four countries of the former Soviet Union, Germany, and the US. Human Resources Development International, 5(1), 99117.

Bass, B. M. (1990). From transactional to transformational leadership: Learning to share the vision. Organization Dynamics, 18(3), 19-36.

Bass, B. M., \& Avolio, B. J. (2004). Multifactor Leadership Questionnaire Manual. ( $3^{\text {rd }}$ Edition). Manual and Sampler Set. Redwood City, CA, EE.UU.: Mind Garden Inc.

Bass, B. M., \& Avolio, B. J. (2000). Effects on platoon readiness of transformational/transactionplatoon leadership. Final Report. (Contract DASW01-96K-0008, U.S. Army Research Institute for the Behavioral and Social Sciences, March 2,000).

Bhaskaran, S., \& Sukumaran, N. (2007). National culture, business culture and management practices: Consequential relationship? Cross Culture Management: An International Journal, 14(7), 54-76.

Byrne G. J., \& Bradley, F. (2007). Culture's influence on leadership efficiency: How personal and national cultures affect leadership style. Journal of Business Research, 60(2), 168-175.

Cullen, J. B., \& Parboteeah, K. P. (2005). Multinational Management: A Strategic Approach. ( $3^{\text {rd }}$ Edition). Ohio: South Western, Thompson Learning.

Day, D. V., \& Lord, R. G. (1988). Executive leadership and organizational performance: Suggestions for a new theory and methodology. Journal of Management, 14(3), 453-464.

Elenkov, D. (1998). Can American management concepts work in Russia? A cross-cultural comparative study. California Management Review, 40(4), 133-156. 
GMAC Global Relocation Services. (2005). Global Relocation Trends: 2005 Survey Report. Recuperado de www.gmacglobalrelocation. com.

Hill, C. (2007). Negocios Internacionales: Competencia en el mercado global. (6ta Edición). New York: Irwin-McGraw-Hill.

Hofstede, G. (2005). Cultures and Organizations: Software of the Mind. $\left(2^{\text {nd }}\right.$ Edition). New York: McGraw Hill.

Hofstede, G. (1980). Culture's consequences: International differences in work-related values. Beverly Hills, CA: Sage Publications.

House, R. J., Chhokar, J. S., \& Brodbeck, F. C. (2004). Culture, Leadership, and Organizations: The Globe Study of 62 Societies. Thousand Oaks, CA: Sage Publications.

House, R. J., Hanges, P. J., Ruiz-Quintanilla, A. S., Dorfman, P. W., Javidan, M., Dickson, M. W., Gupta, V., Global Research Team. (1999). Cultural Influences in Leadership and Organizations: Project GLOBE. In W. H. Mobley, M. J. Gessner, V. Arnold, (eds.), Advances in Global Leadership, Vol. 1. Stamford, Connecticut: JAI Press.

Howell, J. \& Costley, D. (2001). Understanding Behaviors for Effective Leadership. Upper Saddle River, New Jersey: Prentice Hall.

Jung, D., Bass, B., \& Soski, J. (1995). Bridging leadership and culture: a theoretical consideration of transformational leadership and collectivistic cultures. The Journal of Leadership Studies, 2(4), 3-18.

Kustin, R., \& Jones, R. (1995). The influence of corporate headquarters on leadership styles in Japanese and US subsidiary companies. Leadership EO Organization Development Journal, 16(5), 1115.

Lebrón-Rolón, A. (2008). Cultural impact on management styles: A crosscultural study between Puerto Rico and Dominican Republic. Disertación doctoral no publicada. Universidad del Turabo.

Lenartowicz, T., \& Johnson, J. P. (2003). A cross-national assessment of the values of Latin America managers: contrasting hues or shades of gray? Journal of International Business Studies, 34(3), 266-281.

López, H. (2006). Estilos de liderazgo que los directores escolares, exhiben con su conducta administrativa y su relación con el nivel de desarrollo 
como comunidades de aprendizajede las escuelas elementales públicas de Puerto Rico. Disertación doctoral no publicada. Universidad Interamericana de Puerto Rico.

Moro, F. (2005). A cross-cultural comparison of leadership skills across the United States, the United Kingdom and Italy: A quantitative design using internet technology. Disertación doctoral no publicada. Texas A\&M University.

Niedziolek, R. C. (2005). National culture and work related values of Puerto Rico. Disertación doctoral no publicada. Capella University.

Punnett, B. J., Dick-Forde, E., \& Robinson, J. (2006). Effective management and culture: An analysis of three English-speaking Caribbean countries. Journal of Eastern Caribbean Studies, 31 (2), 44-71.

Rentas Rivera, E. J. (2009). Estilos de liderazgo transformacional y transaccional y su impacto en la cultura organizacional de una institución de educación superior en Puerto Rico. Disertación doctoral no publicada. Universidad Interamericana de Puerto Rico.

Robbins, S. P. (2004). Comportamiento organizacional. (10ma Edición). México: Pearson, Prentice Hall.

Robbins, S., \& Coulter, M. (2008). Management. (10 ${ }^{\text {th }}$ Edition). New Jersey: Prentice Hall.

Robbins, S. P., \& Decenzo, D. A. (2001). Fundamentos de administración. (3ra Edición). México: Pearson Educación.

Rodrigues, C. (1990). The situation and national culture as contingencies for leadership behavior: Two conceptual models. Advances in International Comparative Management, 5, 51-68.

Schriesheim, C. A., \& Neider, L. L. (1996). Path-goal leadership theory: The long and winding road. Leadership Quarterly, 7(3), 317-321.

Thomany, F. (2009). An examination of Latino/a American chief executive officer's leadership styles. Disertación doctoral no publicada. Howard University.

Thomas, D. C., \& Au, K. (2002). The effect of cultural differences on behavioral responses to low job satisfaction. Journal of International Business Studies, 33(2), 309-326. 
Vargas, A. M. (2005). Employees' perceived influence of ethnicity of their managers' management style. Tesis de maestría no publicada. California State University.

Vázquez Cruz, Y. (2007). La estructura organizacional, los estilos de liderazgo de los administradores y la efectividad de los programas de educación continua de las universidades privadas de Puerto Rico. Disertación doctoral no publicada. Universidad Interamericana de Puerto Rico.

Vélez Santiago, B. (2008). Estilos de liderazgo de los administradores, su eficacia y el impacto en los niveles de satisfacción y esfuerzo extra de los subalternos. Disertación doctoral no publicada. Universidad Interamericana de Puerto Rico.

Woo, H.S., Wilson, D., \& Liu, J. (2001). Gender impact on Chinese negotiation: some key issues for western negotiators. Women in Management Review, 16(7), 349-356.

Yukl, G. (2010). Leadership in Organizations. ( $7^{\text {th }}$ Edition). New Jersey: Pearson Prentice Hall.

Zagorsek, H. (2005). National culture and usage of transformational leadership practices in six countries. Conference Proceedings: 2005 International Conference Enterprise in Transition, p. 981-1,000. Recuperado el 15 de marzo de 2009 de EBSCO database.

Zagorsek, H., Jaklic, M., \& Stough, S. J. (2004). Comparing Leadership Practices between the United States, Nigeria, and Slovenia: Does culture matter? Cross Cultural Management, 11(2), 16-34. 